\title{
International Journal of Chromatography and Separation Techniques
}

\section{Do Not Underestimate the Good Old Separation Methods used for Protein Isolation}

\author{
Daniela Novick $^{*}$ \\ Molecular Genetics, the Weizmann Institute of Science, Israel
}

"Corresponding author: Daniela Novick, Molecular Genetics, the Weizmann Institute of Science, Israel. Tel: +972545292414; Email: daniela.novick@weizmann.ac.il

Citation: Novick D (2017) Do Not Underestimate the Good Old Separation Methods used for Protein Isolation. Int J Chromatogr Sep Tech: IJCST-110. DOI: 10.29011/IJCST-110.000010

Received Date: 13 September, 2017; Accepted Date: 25 September, 2017; Published Date: 2 October, 2017

\section{Commentary}

In the era of bioinformatics, big data and high throughput techniques it is quite tempting to forget the advantage of the good old techniques such as affinity chromatography, in the search of yet unknown protein molecules. Not so long ago this very efficient and straightforward technique yielded in quite a short time numerous bioactive molecules thus opening new fields of basic research. And I specifically refer to ligand affinity chromatography. This type of separation is based on unique interaction between the target analyte and a ligand coupled covalently to a resin [1,2]. It is a simple, rapid, selective, and efficient purification procedure of proteins. Tens of thousands fold purification is achieved in one step, which is crucial when an extremely rich sources of naturally occurring proteins such as human urine or plasma or spinal fluid are used [3].

The biological activity of the isolated proteins is retained thus function is revealed concomitantly with the isolation. Prior to the completion of the genome project this method facilitated rapid and reliable cloning of the corresponding gene. Upon completion of this project, a partial protein sequence is enough for retrieving its complete mRNA and hence its complete protein sequence. This method is indispensable for the isolation of both expected (e.g. soluble receptors) but mainly unexpected, unpredicted and very much surprising binding proteins found by serendipity. Practically, no other approach would yield the latter [4]. In the group of expected such proteins are the two receptors for TNF $[5,6]$ originally named TNF binding proteins (TBPI and TBPII), the Type I interferon receptor [7], the Type II interferon receptor and the IL-6 receptor [8]. The unexpected group of proteins include the unique IL-18 Binding Protein (IL-18BP) [9], the IL-32 binding protein- Proteinase 3 [10] and the heparanase binding protein, the resistin [11].
In the case of TBPI, the ligand affinity chromatography was a onestep purification procedure, compared to three inefficient purification steps used beforehand to purify the TBPI (CM-Sepharose, cation exchange HPLC, anion exchange HPLC) [5]. Moreover, this affinity column made all the difference since it yielded two TNF binding proteins, the TBPI and the TBPII. The TBPII [6] would have never been discovered by the multistep chromatographic procedure used to purify the TBPI, since its chemistry is different from that of TBPI and it is not retained by the first purification column used for TBPI, the CM-sepharose. Type I interferon was discovered in 1957. Note that it took 37 years to isolate its receptor and not before ligand affinity chromatography was employed [7], though many laboratories all over the world made attempts to isolate it all these years. The discovery of the soluble Type I interferon receptor enabled the identification of its cell-surface counterpart, the ligand binding chain of Type I interferon receptor, later on named IFNAR2.

In our hands, a search for the IL-18 receptor failed but the binding protein that was found instead was a big reward. Encoded by a separate gene, IL-18BP [9] deviates from the classical definition of soluble receptors, namely, it does not correspond to the extracellular ligand-binding domain of the IL-18 receptor but rather could be added as a member of a separate family of rare secreted proteins that bind the same ligand as canonical soluble receptors do, but are encoded by a different gene (e.g. osteoprotegerin, [12]). The exceptionally high affinity of this IL-18BP decoy protein to its target protein, the IL-18 [13], promised its success as a drug that needs no modifications to prolong its half- life time.

This characteristic had already been revealed during the process of its purification, since it smeared though harsh elution conditions were used to take it off the ligand affinity column instead of 
coming off as a sharp peak. Yet additional quite surprising proteins discovered using ligand affinity chromatographies include the enzyme Proteinase 3 (PR3) and resistin. PR3 was isolated upon a search for IL-32 receptor and named IL-32 binding protein [10]. PR3, a neutrophil granule serine protease, is the major antigen for autoantibodies in the systemic vasculitic disease, Wegener's granulomatosis. This disease can be fatal and its treatment requires longterm immunosuppressant. The linkage to IL-32, found by serendipity, may one day shed light on the mechanism of action of this disease.

The pro-inflammatory cytokine resistin was isolated in an attempt to isolate the heparanase receptor and was named heparanase binding protein [11]. Resistin, initially described as a rodent adipokine, is predominantly macrophage-derived in humans and thought to link between inflammation, metabolic diseases and possibly tumorigenesis. Heparanase, among others, is strongly implicated in cancer metastasis and is up-regulated in essentially all human tumors examined. Though resistin and heparanase may have some tangential biological functions, the mechanism of the observed unexpected association between those two remains obscure.

Years later a number of these proteins translated into drugs. It was the TBPII, the second soluble receptor for TNF, namely the one which was isolated by serendipity that became a blockbuster drug Etanercept $\left(\right.$ Enbrel $\left.^{\mathrm{TM}}\right)$. It is since then prescribed to millions of patients to treat rheumatoid arthritis, psoriasis and other chronic inflammatory conditions. The use of IL-18BP in particular conditions is an example for personalized medicine. In 2015, on a compassionate basis, IL-18BP (Tadekinig alfa ${ }^{\circledR}$ ) saved a life of a baby born with a mutation in the inflammasome [14]. This NLRC4 mutation causes an over expression of IL-18 and results in what is known as Macrophage Activating Syndrome (MAS). MAS is a life-threatening sepsis-like condition and is also a complication of cancer, autoimmune diseases and inflammation. IL-18BP is currently in phase III clinical studies for MAS, Hemophagocytic Lymphohistiocytosis (HLH) and the systemic autoinflammatoryStill's disease.

The concept of "Proteome" was introduced more than 10 years ago, followed by large-scale studies of protein expression, localization, activities, and interactions, leading to extensive research and technology development. Proteomics is expansively applied in many areas, ranging from basic research, various disease and tumor diagnoses and biomarker discovery, to therapeutic applications. Several proteomics approaches have been developed for protein separation and identification and for the characterization of protein function and structure. Affinity chromatography and mass spectrometry are among the indispensable methods that enabled this meteoric progress.

\section{References}

1. Hage DS, Matsuda R (2015) Affinity chromatography: a historical perspective. Methods Mol Biol 1286: 1-19.

2. Cuatrecasas P, Wilchek M., Anfinsen CB (1968) Selective enzyme purification by affinity chromatography. Proc Natl Acad Sci U S A 61: 636-643.

3. Novick D, Rubinstein M (2007) The tale of soluble receptors and binding proteins: from bench to bedside. Cytokine Growth Factor Rev 18: 525-533.

4. Novick D Rubinstein M (2012) Ligand affinity chromatography, an indispensable method for the purification of soluble cytokine receptors and binding proteins. Methods in Molecular Biol 820: 195-214.

5. Engelmann H, Aderka D, Rubinstein M, Rotman D, Wallach D (1989) A tumor necrosis factor-binding protein purified to homogeneity from human urine protects cells from tumor necrosis factor toxicity. $\mathrm{J} \mathrm{Bi}$ olChem 264: 11974-11980.

6. Engelmann H, Novick D, Wallach D (1990) Two tumor necrosis factorbinding proteins purified from human urine. Evidence for immunological cross-reactivity with cell surface tumor necrosis factor receptors. J Biol Chem 265: 1531-1536.

7. Novick D, Cohen B, Rubinstein M (1994) The human interferon alpha/ beta receptor: characterization and molecular cloning. Cell 77: 391400.

8. Novick D, Engelmann H, Wallach D, Rubinstein M (1989) Soluble cytokine receptors are present in normal human urine. J Exp Med 170: 1409-1414.

9. Novick D, Kim SH, Fantuzzi G, Reznikov LL, Dinarello CA, et al. (1999) Interleukin-18 binding protein: a novel modulator of the Th1 cytokine response. Immunity 10: 127-136.

10. Novick D, Rubinstein M, Azam T, Rabinkov A, Dinarello CA, et al. (2006) Proteinase 3 is an IL-32 binding protein. Proc Natl Acad Sci U S A 103: 3316-3321.

11. Novick D, Barak S, Ilan N, Vlodavsky I (2014) Heparanase interacts with resistin and augments its activity. PLoS One 9: e85944.

12. Simonet WS, Lacey DL, Dunstan CR, Kelley M, Chang MS, et al. (1997) Osteoprotegerin: a novel secreted protein involved in the regulation of bone density. Cell 89: 309-319.

13. Kim SH, Eisenstein M, Reznikov L, Fantuzzi G, Novick D, et al. (2000) Structural requirements of six naturally occurring isoforms of the IL18 binding protein to inhibit IL18. Proc Natl AcadSci U S A 97: 11901195.

14. Canna SW, Girard C, Malle L, de Jesus A, Romberg N, et al. (2017) Life- threatening NLRC4-associated hyperinflammation successfully treated with IL-18 inhibition. J Allergy Clin Immunol 139: 1698-1701. 